\title{
Spectroscopy of mechanical dissipation in micro-mechanical membranes
}

\author{
Andreas Jöckel, ${ }^{1}$ Matthew T. Rakher, ${ }^{1}$ Maria Korppi, ${ }^{1}$ Stephan Camerer, ${ }^{2}$ David Hunger, ${ }^{2}$ \\ Matthias Mader, ${ }^{2}$ and Philipp Treutlein ${ }^{1, a)}$ \\ ${ }^{1}$ Departement Physik, Universität Basel, CH-4056 Basel, Switzerland \\ ${ }^{2}$ Max-Planck-Institut für Quantenoptik and Fakultät für Physik, Ludwig-Maximilians-Universität, \\ 80799 München, Germany
}

(Received 12 August 2011; accepted 14 September 2011; published online 5 October 2011)

\begin{abstract}
We measure the frequency dependence of the mechanical quality factor $(Q)$ of $\mathrm{SiN}$ membrane oscillators and observe a resonant variation of $Q$ by more than two orders of magnitude. The frequency of the fundamental mechanical mode is tuned reversibly by up to $40 \%$ through local heating with a laser. Several distinct resonances in $Q$ are observed that can be explained by coupling to membrane frame modes. Away from the resonances, the background $Q$ is independent of frequency and temperature in the measured range. (C) 2011 American Institute of Physics. [doi:10.1063/1.3646914]
\end{abstract}

Micro-mechanical membrane oscillators are currently investigated in many optomechanics experiments, where lasers and optical cavities are used for cooling, control, and readout of their mechanical vibrations. ${ }^{1-4}$ Applications lie in the area of precision force sensing and in fundamental experiments on quantum physics at macroscopic scales. ${ }^{5}$ The quality factor $Q$ of the mechanical modes of the membranes is a key figure of merit in such experiments. However, the origin of mechanical dissipation limiting the attainable $Q$ is not completely understood and a subject of intense research. ${ }^{6-11}$

Here, we report an experiment in which we observe a variation of $Q$ by more than two orders of magnitude as a function of the fundamental mode frequency of a SiN membrane. Several distinct resonances in $Q$ are observed that can be explained by coupling to mechanical modes of the membrane frame. ${ }^{7,10}$ The frequency of the membrane modes is tuned reversibly by up to $40 \%$ through local heating of the membrane with a laser. This method of frequency tuning has the advantage that the frequency dependence of $Q$ can be studied with a single membrane in situ, resulting in a detailed spectrum of the coupling to the environment of this particular mode. Other methods that compare $Q$ between various structures of different sizes have to rely on the assumption that the environment of these structures is comparable. ${ }^{11}$

We investigate "low-stress" SiN membranes ${ }^{12}$ that are supported by a Si frame. The frame is glued at one edge to a holder inside a vacuum chamber, see Fig. 1. The eigenfrequencies of a square membrane under tension are

$$
f_{m, n}=\frac{1}{2 l} \sqrt{\frac{S}{\rho}\left(m^{2}+n^{2}\right)},
$$

where $l$ is the side length, $\rho=2.9 \mathrm{~g} / \mathrm{cm}^{3}$ the density, ${ }^{13}$ and $S$ the tensile stress in the membrane. The modes are labeled by the number of anti-nodes $m$ and $n$ along the two dimensions. The stress $S=E\left(l-l_{0}\right) / l_{0}$, where $E$ is Young's modulus, arises in the fabrication process. The SiN membrane is stretched from its equilibrium length $l_{0}$ to the length $l$ of the Si frame.

a)Electronic mail: philipp.treutlein@unibas.ch.
A Michelson interferometer at $852 \mathrm{~nm}$ is used to read out the membrane motion. The interferometer is stabilized by the $\mathrm{DC}$ to $20 \mathrm{kHz}$ part of the photodiode (PD) signal. The incident power on the membrane is $580 \mu \mathrm{W}$ in a diameter of $150 \mu \mathrm{m}$ and the position sensitivity is $1 \times 10^{-14} \mathrm{~m} / \sqrt{\mathrm{Hz}}$. The $>100 \mathrm{kHz}$ part of the signal is fed into a lock-in amplifier with integrated phase locked loop, which measures the membrane amplitude and drives its motion via a piezo (PZT) mounted outside of the vacuum chamber. To tune the membrane frequency, a power stabilized $780 \mathrm{~nm}$ laser is focused onto the membrane to a diameter of $350 \mu \mathrm{m}$. This laser heats the membrane locally in its center. A second method of heating the whole membrane and frame is by a resistive heater (R) in the chamber.

In a first experiment, we demonstrate the tunability of the membrane eigenfrequencies through laser heating. Fig. 2 shows the recorded mode spectrum as a function of heating laser power $P$. The spectra are recorded by Fourier transforming the PD signal. One can see a reversible decrease of all mode frequencies $f_{m, n}$ with $P$.

The decrease in frequency can be attributed to a thermal expansion of the membrane $\Delta l_{0} / l_{0}=\alpha_{0} \Delta T+\alpha_{1} \Delta T^{2}$, where

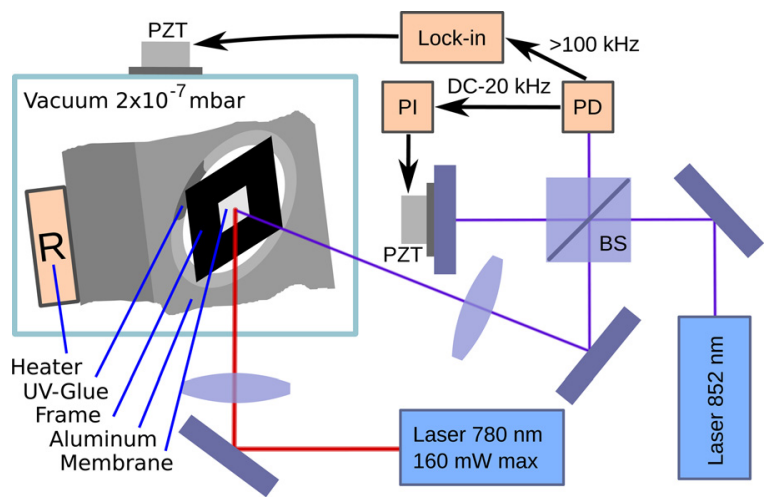

FIG. 1. (Color online) Experimental setup. The SiN membrane in a Si frame is glued at one edge to an aluminum holder inside a room-temperature vacuum chamber. The heating laser (red, thick line) at $780 \mathrm{~nm}$ is power stabilized to $2 \times 10^{-4}$ RMS in a bandwidth of $12 \mathrm{kHz}$ and focused onto the membrane under an angle. The membrane vibrations are read out with a stabilized Michelson interferometer (blue, thin line). The interferometer signal is also used for feedback driving of the membrane with a piezo. 


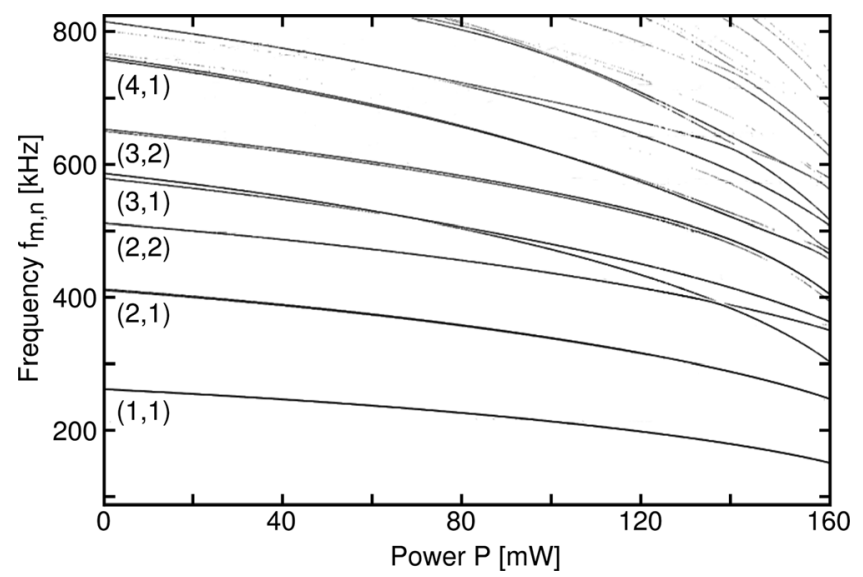

FIG. 2. Mode spectrum $f_{m, n}$ of a membrane $(l=0.5 \mathrm{~mm}$, thickness $t=50 \mathrm{~nm})$ as a function of $P$. At $P=0$, the lowest 13 modes lie within $2 \%$ of the expected frequency. At higher $P$, anticrossings between higher order modes are visible.

$\Delta l_{0}$ is the equilibrium length change and $\alpha_{0}\left(\alpha_{1}\right)$ the first (second) order expansion coefficient for a temperature change $\Delta T$. This reduces the tensile stress by $\Delta S=-E\left(\Delta l_{0} / l_{0}\right)$. In a simple model, assuming a spatially homogeneous and linear temperature change with power $\Delta T=\chi P$, one can describe the power-dependence of the stress as

$$
S=S_{0}-E\left(\Delta l_{0} / l_{0}\right)=S_{0}-E\left(\alpha_{0} \chi P+\alpha_{1} \chi^{2} P^{2}\right) .
$$

A fit of $f_{1,1}(P)$ to the data describes the observed dependence within $\pm 1 \mathrm{kHz}$. For low $P$ we observe a linear shift of $\Delta f_{1,1}=-363 \mathrm{~Hz} / \mathrm{mW}$. We neglect the dependence of $E$ on $\Delta T$ because it is small. ${ }^{14}$ As shown in Table I, the tunability of $f_{1,1}$ depends strongly on the geometry.

In order to extract $\chi$ from the fit, one has to measure $\alpha_{0}$. This is done by heating up the whole sample holder with the resistive heater. In this case, both $l$ and $l_{0}$ change and the difference in the expansion coefficients $\Delta \alpha=\alpha_{f}-\alpha$ of the frame and the membrane determines $S-S_{0}=E \Delta \alpha \Delta T$. Heating the setup by $\Delta T=16 \mathrm{~K}$ and using $^{14} E=260 \mathrm{GPa}$ and $^{15}$ $\alpha_{f}=2.6 \mathrm{ppm} / \mathrm{K}$, one gets $\alpha_{0}=1.6 \mathrm{ppm} / \mathrm{K}, \alpha_{1}=1.3 \times 10^{-8} / \mathrm{K}^{2}$, and $\chi=0.6 \mathrm{~K} / \mathrm{mW}$. This yields an average membrane temperature of $T=100^{\circ} \mathrm{C}$ for $P=160 \mathrm{~mW}$. To model laser absorption in the membrane, we perform a finite element (FEM) simulation of laser heating using a Gaussian beam profile and a heat conductivity $^{13} \kappa=3 \mathrm{~W} / \mathrm{Km}$. From the resulting temperature distribution, we calculate the average membrane temperature for a given absorbed laser power. By comparing with $\chi$, we find an absorption of $1.5 \times 10^{-3}$ at $780 \mathrm{~nm}$, an order of magnitude larger than the absorption in low-stress membranes at $1064 \mathrm{~nm}^{1}$

TABLE I. Summary of measured SiN membrane parameters. $Q_{\max }$ refers to the maximum observed $Q$. Values marked by ${ }^{\star}$ were limited by the available tuning range.

\begin{tabular}{lcccccc}
\hline \hline$l(\mu \mathrm{m})$ & 250 & 500 & 1000 & 1500 & 500 & 500 \\
$t(\mathrm{~nm})$ & 50 & 50 & 50 & 50 & 75 & 100 \\
$S_{0}(\mathrm{MPa})$ & 66.4 & 98.0 & 120 & 78.8 & 114 & 217 \\
$f_{1,1}(\mathrm{kHz})$ & 428 & 260 & 144 & 77.7 & 281 & 387 \\
$\Delta f_{1,1}(\mathrm{~Hz} / \mathrm{mW})$ & -259 & -363 & -68.9 & -49.5 & -89.6 & -10.5 \\
$Q_{\max }\left(10^{5}\right)$ & 3.2 & 10 & 15 & $\star 5.7$ & 10 & ${ }^{\star} 0.37$ \\
\hline \hline
\end{tabular}

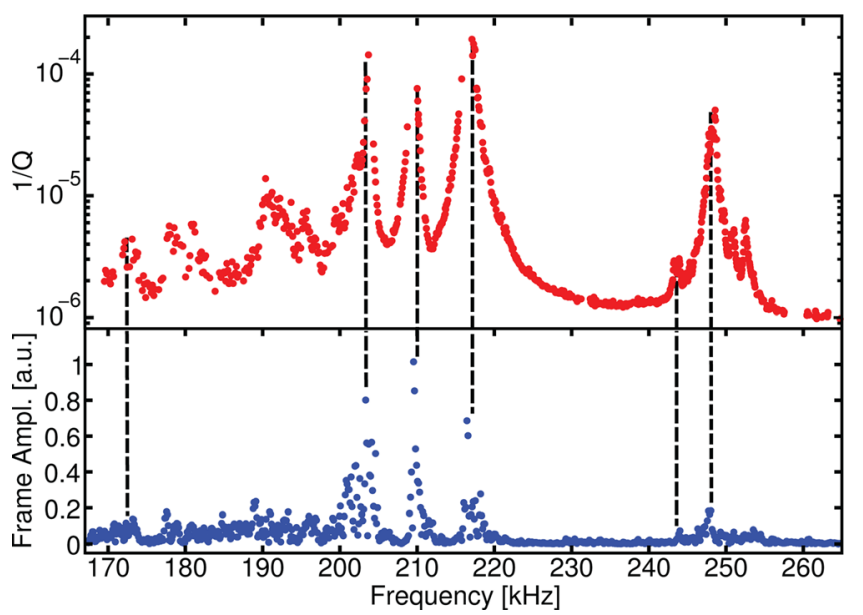

FIG. 3. (Color online) Upper plot: spectrum of membrane dissipation $Q^{-1}\left(f_{1,1}\right)$. Lower plot: vibrations of the frame measured close to the membrane. The resonances in $Q^{-1}\left(f_{1,1}\right)$ coincide with the frame modes.

In a second experiment, we use laser tuning to record a spectrum of the quality factor $Q$ of the fundamental mode as a function of $f_{1,1}$. We measure the decay time $\tau$ of the membrane amplitude in ring-down measurements after driving it to $\approx 0.5 \mathrm{~nm}$. The upper plot in Fig. 3 shows the dissipation $Q^{-1}=1 / \pi f \tau$. We observe distinct resonances, changing $Q$ by more than two orders of magnitude. To show that the spectrum directly depends on $f_{1,1}$, the heating laser is pointed off center such that a different dependence $f_{1,1}(P)$ results, see Fig. 4(a). The dependence $Q^{-1}\left(f_{1,1}\right)$ is unchanged, showing that $Q$ only indirectly depends on $P$. The resonances in $Q$ can be attributed to coupling of the membrane mode to modes of the frame. To prove this, the interferometer is pointed onto the frame next to the membrane and the amplitude response to a driving with the piezo is recorded, as shown in the lower plot in Fig. 3. The observed frame modes clearly overlap with the resonances in $Q^{-1}$. If the frame is heated with the resistive heater, we observe a shift in the resonances in $Q^{-1}\left(f_{1,1}\right)$, as shown in Fig. 4(b). We attribute this to a shift of the frame modes due to thermal expansion and decreasing Young's modulus.

All these measurements prove that the coupling to frame modes is responsible for the observed behavior of $Q$. A FEM simulation of the frame modes shows roughly the right density of modes in the frequency range of interest. As the eigenfrequencies depend strongly on the exact mounting,
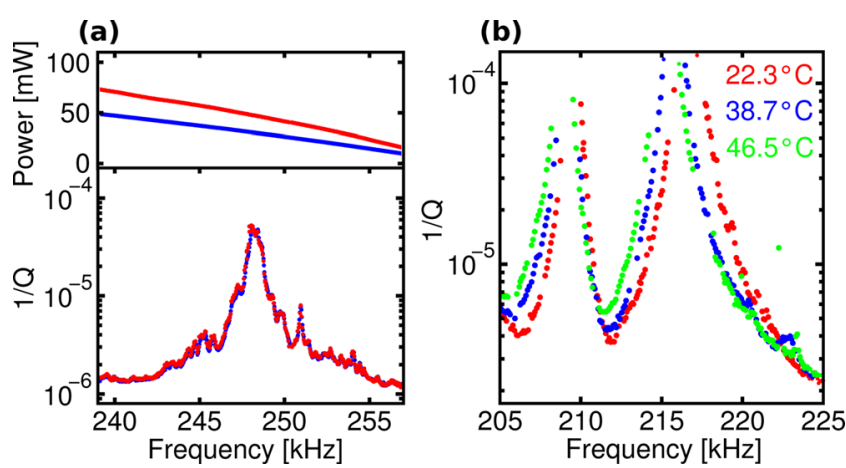

FIG. 4. (Color online) (a) $f_{1,1}(P)$ and $Q^{-1}\left(f_{1,1}\right)$ for different heating laser positions (membrane center: blue (lower line), off-center: red (upper line)). (b) $Q^{-1}\left(f_{1,1}\right)$ for different sample temperatures. 


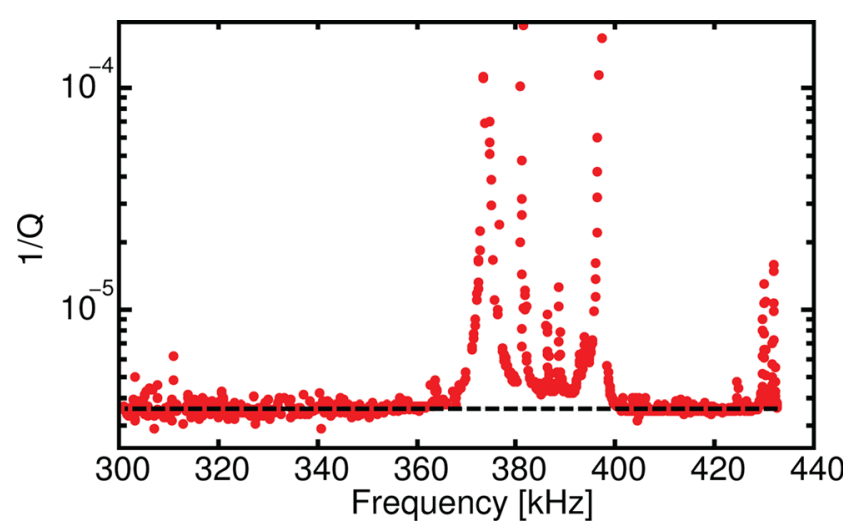

FIG. 5. (Color online) Spectrum of membrane dissipation $Q^{-1}\left(f_{1,1}\right)$ for another membrane $(l=250 \mu \mathrm{m}, t=50 \mathrm{~nm})$.

dimensions, and Young's modulus of the frame, it is difficult to model them quantitatively.

For stoichiometric $\mathrm{Si}_{3} \mathrm{~N}_{4}$ "high-stress" membranes $\left(S_{0}=980 \mathrm{MPa}\right)$, we observe a much weaker dependence of the mode frequencies on $P$. The measurements indicate that absorption of $780 \mathrm{~nm}$ light is lower by two orders of magnitude compared to the "low-stress" membranes. This is of importance for experiments coupling such membranes to atomic systems. ${ }^{3}$ Using the limited tuning range of the resistive heater, we also observe a change of $Q$ with frequency in highstress membranes. This shows that coupling to frame modes is also important in this case. The highest measured $Q$ is $4 \times 10^{6}$ for a high-stress membrane with $l=1.5 \mathrm{~mm}$ and $t=50 \mathrm{~nm}$.

Besides coupling to frame modes, the frequency dependence of other dissipation mechanisms is of interest. Fig. 5 shows the dissipation spectrum of another low-stress membrane. Away from the resonances, we observe a constant baseline $Q_{\max }$, indicating that other dissipation mechanisms are independent of $f(S)$ and $T$ within our tuning range. This is in contrast to what has been observed in SiN strings. ${ }^{6,9}$ For membranes where a constant baseline was observed, $Q_{\max }$ increases with $l$, see Table I. We also studied higher order modes up to $(2,2)$ and find approximately the same $Q_{\max }$, in contrast to other membrane experiments. ${ }^{10}$ This could be due to the different frame geometry and mounting. In our case, the frame is a relatively small resonant structure with eigenmodes at distinct frequencies. This can be exploited to reduce clamping loss by tuning the membrane frequency to a gap between frame modes, analogous to the recently demonstrated phononic bandgap shielding. ${ }^{16}$
In conclusion, we presented a precise method for lasertuning of micro-mechanical membrane oscillators and used it for spectroscopy of mechanical dissipation. Resonances in the dissipation were observed and explained as coupling to localized frame modes. Other dissipation mechanisms were found to be independent of membrane frequency and temperature in the measured range. Our laser tuning technique could be extended to stoichiometric $\mathrm{Si}_{3} \mathrm{~N}_{4}$ membranes by using a laser with smaller wavelength and, thus, higher absorption. ${ }^{17}$ This would allow further investigation of the differences between low-stress and stoichiometric membranes. Moreover, it could be useful in finding optimal frame geometry and mounting conditions to circumvent clamping loss.

We acknowledge helpful discussions with I. WilsonRae, M. Aspelmeyer, K. Hammerer, and T. W. Hänsch. Work supported by the EU project AQUTE and the NCCR Nanoscale Science.

${ }^{1}$ J. D. Thompson, B. M. Zwickl, A. M. Jayich, F. Marquardt, S. M. Girvin, and J. G. E. Harris, Nature 452, 72 (2008).

${ }^{2}$ D. J. Wilson, C. A. Regal, S. B. Papp, and H. J. Kimble, Phys. Rev. Lett. 103, 207204 (2009).

${ }^{3}$ S. Camerer, M. Korppi, A. Jöckel, D. Hunger, T. W. Hänsch, and P. Treutlein, e-print arXiv:1107.3650.

${ }^{4}$ D. Friedrich, H. Kaufer, T. Westphal, K. Yamamoto, A. Sawadsky, F. Y. Khalili, S. Danilishin, S. Goßler, K. Danzmann, and R. Schnabel, New J. Phys. 13, 093017 (2011)

${ }^{5}$ T. J. Kippenberg and K. J. Vahala, Science 321, 1172 (2008); I. Favero and K. Karrai, Nat. Photonics 3, 201 (2009); D. Hunger, S. Camerer, M. Korppi, A. Jöckel, T. W. Hänsch, and P. Treutlein, e-print arXiv:1103.1820.

${ }^{6}$ S. S. Verbridge, D. F. Shapiro, H. G. Craighead, and J. M. Parpia, Nano Lett. 7, 1728 (2007).

${ }^{7}$ I. Wilson-Rae, Phys. Rev. B 77, 245418 (2008).

${ }^{8}$ D. R. Southworth, R. A. Barton, S. S. Verbridge, B. Ilic, A. D. Fefferman, H. G. Craighead, and J. M. Parpia, Phys. Rev. Lett. 102, 225503 (2009).

${ }^{9}$ Q. P. Unterreithmeier, T. Faust, and J. P. Kotthaus, Phys. Rev. Lett. 105, 027205 (2010).

${ }^{10}$ I. Wilson-Rae, R. A. Barton, S. S. Verbridge, D. R. Southworth, B. Ilic, H. G. Craighead, and J. M. Parpia, Phys. Rev. Lett. 106, 047205 (2011).

${ }^{11}$ G. D. Cole, I. Wilson-Rae, K. Werbach, M. R. Vanner, and M. Aspelmeyer, Nature Commun. 2, 231 (2011).

${ }^{12}$ Norcada, Inc.; frame dimensions: $l=5 \mathrm{~mm}, t=200 \mu \mathrm{m}$.

${ }^{13}$ B. Zink and F. Hellman, Solid State Commun. 129, 199 (2004).

${ }^{14}$ W.-H. Chuang, T. Luger, R. Fettig, and R. Ghodssi, J. Microelectromech. Syst. 13, 870 (2004).

${ }^{15}$ K. G. Lyon, G. L. Salinger, C. A. Swenson, and G. K. White, J. Appl. Phys. 48, 865 (1977).

${ }^{16}$ T. P. M. Alegre, A. Safavi-Naeini, M. Winger, and O. Painter, Opt. Express 19, 5658 (2011).

${ }^{17}$ W. C. Tan, S. Kobayashi, T. Aoki, R. E. Johanson, and S. O. Kasap, J. Mater. Sci.: Mater. Electron. 20, S15 (2009). 